\title{
A Real Time Non Intrusive Accident Avoidance System
}

\author{
Syed Imran Ali, PhD \\ HOD,CSE \\ Sagar Institute of Science, \\ Technology and Research \\ Bhopal,India
}

\author{
Zohaib Khan \\ Research Scholar \\ Sagar Institute of Science, \\ Technology and Research
}

\author{
Sameer Jain \\ Assistant Professor (SOGM) \\ NICMAR,Pune,India
}

\begin{abstract}
This paper presents a real time system for accident avoidance system based on drowsiness detection.

The proposed system uses the time-efficient image processing techniques to measure eyes closer count, blinking rate of eye and user yawning as the parameters to conclude drowsiness in the user.

The user could be any person like a computer operator controlling heavy machineries like cranes or performing time, operating critical operations on distant machines, hands free interaction with computational devices/machines, or handling critical operations like air traffic controlling etc. Same system can also be employed to detect and notifying the driver vigilance level and hence to avoid possibility of road accidents.

The proposed system continuously captures the image of the user using web camera and detects face region, then focuses on eyes and lips using efficient image processing techniques to monitor their behavior. If abnormality either in behavior of eyes or mouth is detected, it indicates that the user is falling asleep therefore fatigue is concluded and a warning alarm is generated.
\end{abstract}

\section{General Terms}

Drowsiness. Non Intrusive accident avoidance systems,

\section{Keywords}

Face detection, eye blinking rate, eye closer count, yawning, edge density, threshold value

\section{INTRODUCTION}

Significant efforts are found in the literature for developing an active safety system for reducing the number of accidents due to reduced attentiveness. Drowsiness detection in the user can be generally divided into the following categories:

1. Sensing of physiological characteristics of the user,

2. Sensing of user operation,

3. Sensing of machine response,

4. Monitoring the response of the user.

Among these methods, the best techniques in terms of accuracy are the ones that are based on Human physiological phenomena [1].

They are implemented either in measuring changes in physiological signals, such as brain waves, heart rate, and eye blinking or by measuring physical changes such as sagging posture, leaning of the user's head and the open/closed states of the eyes [1].

The first technique, while most accurate, is not realistic, since sensing electrodes would have to be attached directly onto the user's body, and hence be annoying and distracting to the user. In addition, long time usage would result in perspiration on the sensors, diminishing their ability to sense accurately. The second technique is well suited for real world conditions since it could be non-intrusive by using optical sensors of video cameras to detect changes.

User operation and machine behavior is good indicator of detecting alertness level of user. These are non intrusive ways of detecting drowsiness, but unfortunately are limited to machine type and user conditions. The final technique for detecting drowsiness is by monitoring the response of the user. This involves periodically requesting the user to send a response to the system to indicate alertness. The problem with this technique is that it will eventually become tiresome and annoying to the user.

The proposed system is altogether different from discussed techniques. It is based on eyes closer count (blinking rate), duration of eye closure (nap detection) and yawning detection of the user. By monitoring the eyes and mouth, it is believed that the symptoms of user fatigue can be detected early enough to avoid an accident. Blinking rate of normal eye is 45 times per minute. In drowsiness state, eye blinking rate either increases or decreases by normal rate. Similarly duration of eye closure is also a significant parameter in detecting somnolence because in drowsiness state eye closure duration increases to 3-4 seconds (micro sleeps). But it is difficult to predict alertness level based only on eyes functioning because previous researches have proven that eye detection has several limitations like if user is wearing eyeglasses then eye detection becomes a problem[1],[2],[3]. Also, eye detection is difficult for users having smaller eyes.

Moreover, if we only look at the number of consecutive frames where the eyes are closed, then at that point it may be too late to issue the warning. To overcome this, proposed system also considers yawning detection as a second parameter to detect somnolence. Yawning is a very good indicator to detect drowsiness state [1] and if yawning is detected then warning signal may be issued early enough to avoid accident.

This system has been designed to be deployed in real time environment at very less cost because it does not require additional hardware. Previous works requires extra hardware like frame grabbers [3], infra red camera [2] to detect drowsiness which increases the cost of system to a great extent. Also, it does not require additional surrounding light sources as required by previous research [3]. Proposed system works efficiently even in the presence of different illumination sources in the background, unlike the previous research [3] which requires that there should be dark background behind the user. 
In addition to these qualities, proposed application is very light weight (space and time efficient), does not eat up much CPU execution time.

Ideally any software proposed to help us should not affect our normal course of interaction with the system otherwise the usability and acceptability gets hampered adversely like some antivirus software make the system responding too slow to the user and even some times disturb the user with their annoying pop ups sometimes disturbs our normal functioning. This system has been proposed by keeping this point into consideration.

Therefore, proposed system identify fatigue accurately and efficiently while incurring minimized time complexity, space complexity, cost and overheads of using it.

\section{PROPOSED ALGORITHM}

1. Take new image (RGB) and convert it into Grayscale image.

2. Find the edges of the image using Sobel Filters.

3. Identify the face within the image.

4. Cut the image of face into two halves horizontally to get image of eyes and mouth separately.

5. For the image of eyes do the following:

a. Calculate Edge Density.

b. Compare it with threshold value

(i) If edge density < threshold value, then generate alarm.

(ii) If edge density > threshold value, then go to step 1.

6. For the image of mouth do the following:

a. Calculate Edge Density.

b. Compare it with threshold value

(i) If edge density $>$ threshold value, then generate alarm.

(ii) If edge density < threshold value, then go to step 1 .

7. Calculate Blinking rate of eyes. If it founds to be abnormal then generate alarm, else go to step1.

\subsection{Converting the input RGB image into Gray image.}

Since RGB image takes 24 bits $(8 \times 3)$ while gray scale image takes only 8 bits to store and we are interested in developing a time efficient system, therefore after capturing the image (RGB image) it is converted into gray scale image. Intensity of an image is the average of the three color elements, so the gray scale image that represent the original color image can be computed as:

$$
\mathrm{I}_{0}=(\mathrm{Ri}+\mathrm{Gi}+\mathrm{Bi}) / 3
$$

Io is the output intensity; $\mathrm{Ri}, \mathrm{Gi}$, and $\mathrm{Bi}$ are the red, green, and the blue element intensity. The formula for more realistic result is by adding different weight for each $R, G$, and $B$ element. We normally percept green color brighter that red color, and red color brighter than blue color. That's why we usually set the weight higher for red and higher for green.

$$
\mathrm{I}_{0}=(0.299 \mathrm{Ri}+0.587 \mathrm{Gi}+0.144 \mathrm{Bi}) / 3
$$

Actually there is no absolute reference for each weight values because it depends on the display technology that might change in the future. The above formula is standardized by NTSC (National Television System Committee), and its usage is common in computer imaging [8].

The MATLAB command used to convert RGB image to grayscale image is:

set(vid,'ReturnedColorSpace','grayscale');

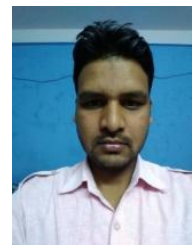

Fig 2.1 RGB and its equivalent grayscale image

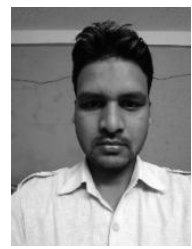

Fig 2.2 Finding the edges of blurred image

To find the edges, Sobel filters have been used.

Sobel edge detection filter algorithm

Two $3 \times 3$ convolution masks are applied to each pixel, one color at a time - one with a horizontal trend and one with a vertical trend. The result of the each convolution is treated a vector representing the edge through the current pixel. If the magnitude of the sum of these two orthogonal vectors is greater than some user-specified threshold, the pixel is marked in black as an edge. Otherwise, the pixel is set to white [5].

\begin{tabular}{|l|l|l|}
\hline \multicolumn{3}{|c|}{ Vertical Kernel } \\
\hline-1 & 0 & +1 \\
\hline-2 & 0 & +2 \\
\hline-1 & 0 & +1 \\
\hline
\end{tabular}

\begin{tabular}{|l|l|l|}
\hline \multicolumn{3}{|c|}{ Horizontal Kernel } \\
\hline-1 & -2 & -1 \\
\hline 0 & 0 & 0 \\
\hline+1 & +2 & +1 \\
\hline
\end{tabular}

Fig. 2.2 shows the edges of image taken after applying Sobel Filter method

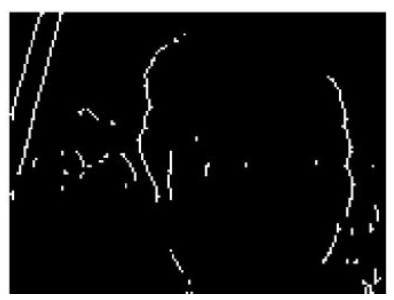

Fig. 2.2 edge detection

\subsubsection{Thresholding}

Once we have computed a measure of edge strength (typically the gradient magnitude), the next stage is to apply a threshold, to decide whether edges are present or not at an image point. The lower the threshold, the more edges will be detected, and the result will be increasingly susceptible to noise, and also to 
picking out irrelevant features from the image. Conversely a high threshold may miss subtle edges, or result in fragmented edges.

If the edge thresholding is applied to just the gradient magnitude image, the resulting edges will in general be thick and some type of edge thinning post-processing is necessary. For edges detected with non-maximum suppression however, the edge curves are thin by definition and the edge pixels can be linked into edge polygon by an edge linking (edge tracking) procedure. On a discrete grid, the non-maximum suppression stage can be implemented by estimating the gradient direction using first-order derivatives, then rounding off the gradient direction to multiples of 45 degrees, and finally comparing the values of the gradient magnitude in the estimated gradient direction.

A commonly used approach to handle the problem of appropriate thresholds for thresholding is by using thresholding with hysteresis. This method uses multiple thresholds to find edges. We begin by using the upper threshold to find the start of an edge. Once we have a start point, we then trace the path of the edge through the image pixel by pixel, marking an edge whenever we are above the lower threshold. We stop marking our edge only when the value falls below our lower threshold. This approach makes the assumption that edges are likely to be in continuous curves, and allows us to follow a faint section of an edge we have previously seen, without meaning that every noisy pixel in the image is marked down as an edge. Still, however, we have the problem of choosing appropriate thresholding parameters, and suitable thresholding values may vary over the image.

\subsection{Face Detection}

From the edges of the image ,next step is to find face among edges. For finding the face, start scanning the image from both ends left as well as right end and marking great intensity changes along y coordinates or vertical direction

Great intensity changes denotes left and right corner of face. Fig. 2.3 shows the graph showing the intensity changes of the image. Face region is found between two highest peaks.

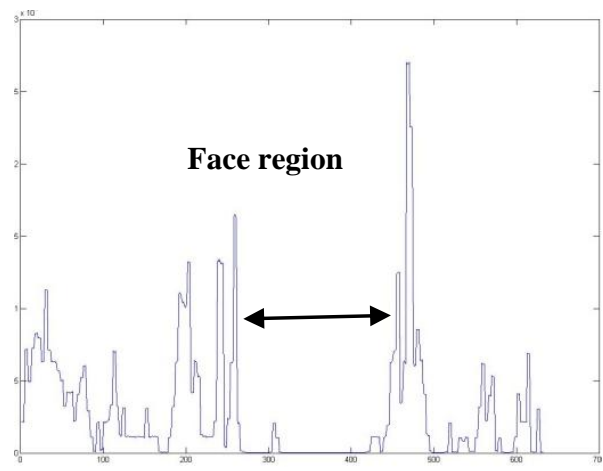

Fig. 2.3 Face detection

\subsection{Cutting the image of face into two halves}

By cutting the image of face into two halves horizontally from the centre,gives the images of eyes and mouth separately.

Now eyes movement and yawning can be monitered independently as the system has two independent images to process as shown in Fig. 2.4
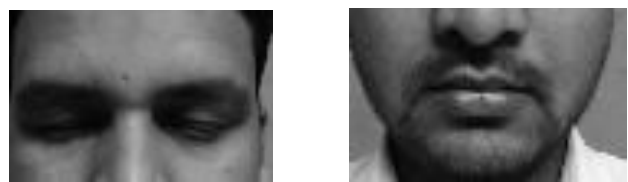

Fig.2.4 Calculation of edge density

Now the edge densty in each image is calculated and then their average is taken to find if eyes are open or closed. If the number of edges are less than the threshold value, it indicates eyes are closed else eyes are open.This is shown in Fig2.6
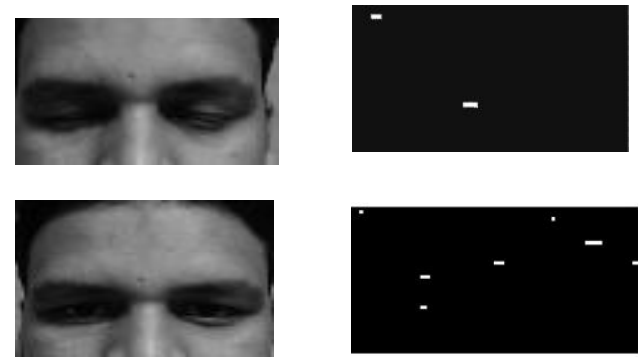

Fig.2.5

If eyes are found to be close for 4-5 consecutive frames then snap is detected and hence warning signal is generated. Similarly, we calculate edge density in image of mouth to detect yawning .If the number of edges are less than the threshold value, it indicates mouth is closed else it is open. This is shown in fig 2.6
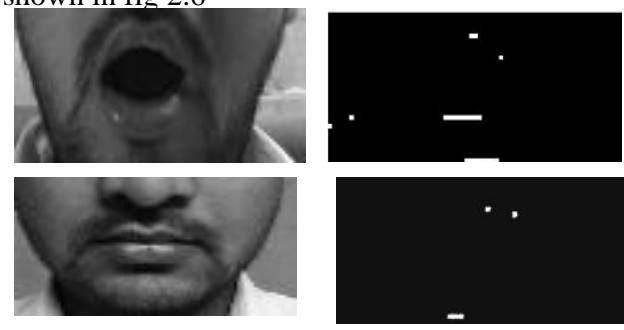

Fig. 2.6

If mouth is found to be open for 3-4 consecutive frames then yawning is detected ,hence warning signal is generated.

\section{RESULTS}

The Proposed system is implemented in real time and work successfully.It was experimented on 6 different people of different demographics and gave high accuracy (more than $90 \%)$.

All the pictures and results used in this paper are obtained from actual implementation of system.

\subsection{When Nap is detected}

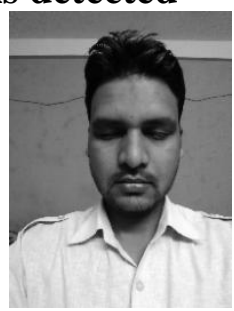




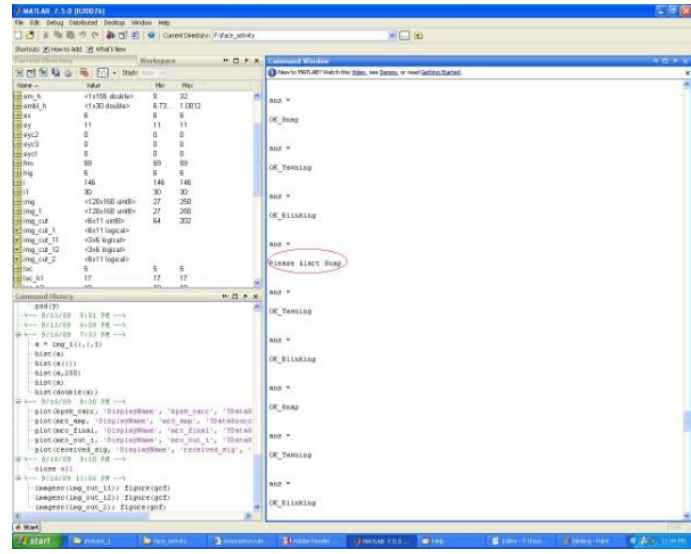

Fig. 2.6 Nap Detection

\subsection{When yawning is detected}

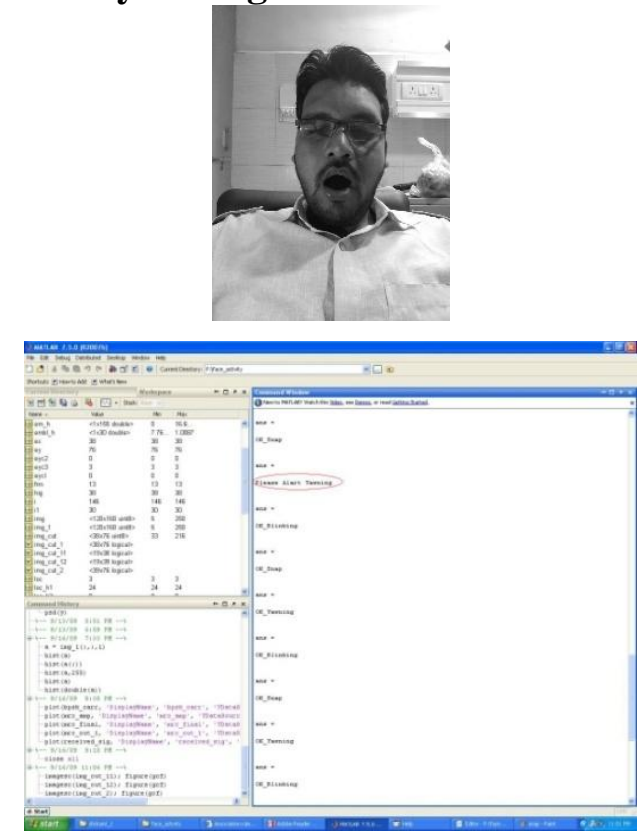

Fig. 2.7 Yawning detection

Case 3: When Blinking Rate is Abnormal

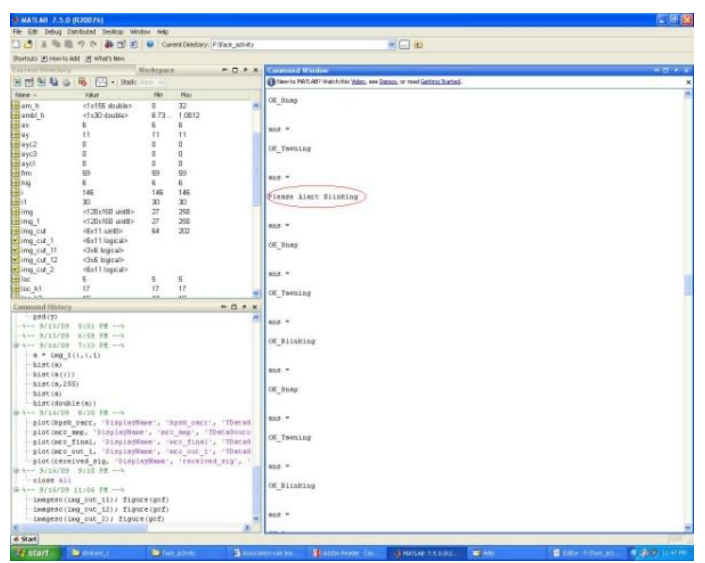

Fig. 2.8 Blinking rate detection

\section{CONCLUSION}

This paper presents a real time non intrusive accident avoidance system based on drowsiness detection. The proposed system is based on eyes closer, blinking rate of eye $\&$ yawning detection of the user. The system continuously captures the image of the subject on site and detects face region, then eyes and lips are detected in the face under consideration to determine if eyes are closed or open, if eyes found to be closed for 4-5 consecutive frames or blinking rate of is found to be abnormal or lips are found to be open for long duration (yawning), for 3-4 consecutive frames then it is concluded that the subject is falling asleep or having state of drowsiness therefore fatigue is detected and a warning alarm issued.

The proposed system can be applied to large number of applications like in vehicles to detect drowsiness level of user, in software industries where developers works continuously for 14-15 hours to detect their vigilance level, for computer operators, operating critical operations on distant machines, hands free interaction with computational devices/machines, controlling heavy machineries like cranes etc.

The proposed system has following advantages

1. Works in real time without requiring any extra hardware, thus reducing the cost of system to a great extent.

2. Works with users wearing eyeglasses or having smaller eyes also

3. Works even when background is not uniform.

4. Computations are very easy and it is a very lightweight system, does not irritate user while using it and does not disturb user in doing his regular work unlike antivirus software.

\section{REFERENCES}

[1] N.G.Narole , Dr.P.R.Bajaj "A Neuro-Genetic System Design for Monitoring User's Fatigue", IJCSNS International Journal of Computer Science and Network Security, VOL.9 No.3, March 2009

[2] K.S.Chidanand Kumar and Brojeshwar Bhowmick "An Application for User Drowsiness Identification based on Pupil Detection using IR Camera.", Innovation lab ,TCS,Kolkata,India.2008

[3] N. Parmar , "Drowsy User Detection System",Engineering Design Project Thesis, Ryerson University,2002.

[4] H.J. Dikkers, Spaans, "Facial Recognition System for User Vigilance Monitoring", Delft University of Technology, Delft, Netherlands, IEEE 2004.

[5] www.jasonokane.com

[6] Singh, Sarbjit and Papanikolopoulos "Monitoring Driver Fatigue Using Facial Analysis Techniques", IEEEIntelligent Transport System Proceedings, , pp. 314-318, 1999

[7] S. Jin , S.-Y. Park - J.-J. Lee "Driver Fatigue Detection using Genetic Algorithm.", 11th International Symposiumon Artificial Life and Robotics, Oita, Japan, January 23-25, 2006

[8] imageprocessingindelphi.blogspot.com 
[9] Yan Tonga, YangWangb, Zhiwei Zhuc, Qiang Jia “ Robust Facial Feature Tracking Under Varying Face Pose And Facial Expression”,,Department of Electrical, Computer, and Systems Engineering, Rensselaer Polytechnic Institute, Troy, NY 12180-3590, USA,2007

[10] Jeffrey Huang and Harry Wechsler " Eye Location Using Genetic Algorithm ", $2^{\text {nd }}$ International Conference on Audio and Video-Based Biometric Person Authentication (AVBPA), Washington, DC, 1999.

[11] www.ostermiller.org.

[12] F. Smach, M. Atri, J. Mitéran and M. Abid "Design Of A Neural Networks Classifier For Face Detection", Journal of Computer Science 2 (3): 257 260, 2006,ISSN 1549-3636, Science Publications, 2006

[13] Weirwille, W.W.. "Overview of Research on Driver Drowsiness Definition and Driver Drowsiness Detection," 14th International Technical Conference onEnhanced Safety of Vehicles, pp 23-26, 1994.

[14] K. Yammamoto and S.Higuchi, J.Soc "Development of drowsiness warning System". Automotive Eng. Japan, pp. 127-133.
[15] J. Fakuda , K. Adachi and M. Nishida "Development of driver's drowsiness detection technology"., Toyota, Tech. Rev.vol .45, pp. 34-40, 1995

[16] R.L.Hus, M.A.Mottaleb and A.K.Jain "Face detection in color images", IEEE Trans. Pattern Analysis and Machine Intell. 24, , pp. 696-706, 2003.

[17] M.Yang, D.J.Kriegman and N.Ahuja "Detecting faces in images: A survey", IEEE Trans. Pattern Analysis and machine Intell.24, pp. 34-58, 2002.

[18] Eriksson, M and Papanikolopoulos, N.P. "Eye-tracking for Detection of Driver Fatigue", IEEE Intelligent Transport System Proceedings, pp 314-319, 1997.

[19] Digital image processing: Rafael C. Gonzalez

[20] Perez, Claudio A. et al. "Face and Eye Tracking Algorithm Based on Digital Image Processing", IEEE System, Man and Cybernetics 2001 Conference, vol. 2 , pp 1178-1188, 2001

[21] Ueno H., Kanda, M. and Tsukino, M. "Development of Drowsiness Detection System", IEEE Vehicle Navigation and Information Systems Conference Proceedings,ppA1-3, 15-20, 1994. 